\title{
Host-microbial interactions in the metabolism of therapeutic and diet-derived xenobiotics
}

\author{
Rachel N. Carmody and Peter J. Turnbaugh \\ FAS Center for Systems Biology, Harvard University, Cambridge, Massachusetts, USA.
}

\begin{abstract}
Our associated microbial communities play a critical role in human health and predisposition to disease, but the degree to which they also shape therapeutic interventions is not well understood. Here, we integrate results from classic and current studies of the direct and indirect impacts of the gut microbiome on the metabolism of therapeutic drugs and diet-derived bioactive compounds. We pay particular attention to microbial influences on host responses to xenobiotics, adding to the growing consensus that treatment outcomes reflect our intimate partnership with the microbial world, and providing an initial framework from which to consider a more comprehensive view of pharmacology and nutrition.
\end{abstract}

\section{Introduction}

The human body is home to a complex microbial ecosystem. The gastrointestinal tract houses the most numerous microbial community (the gut microbiota), consisting of approximately $10^{13}$ microbial cells (1). Collectively, the aggregate genomes of the gut microbiota encode more than 100 times as many unique genes as the approximately 20,000 protein-coding genes found in the human genome and nearly 20,000 gene families (2). Therefore it is not surprising that these genes and their encoded metabolic activities (the gut microbiome) expand host metabolic capabilities. In this Review, we focus on one particular area of critical relevance to clinical practice and investigation: the impact of the gut microbiome on xenobiotics, foreign compounds including therapeutic drugs and diet-derived bioactive compounds (Figure 1). We highlight key studies that illustrate the breadth of microbial influences and the diversity of mechanisms involved, while providing initial evidence that interindividual differences in the human gut microbiome contribute to variations in xenobiotic response. Finally, we discuss how this emerging area of study might be best leveraged to improve medicine in the coming years.

\section{Direct microbial interference with xenobiotics}

Xenobiotic metabolism typically involves the enzymatic conversion of readily absorbed lipophilic compounds into hydrophilic products for excretion. The liver is the major site of these conversions, but relevant biotransformations also occur in the gastrointestinal tract and other tissues (3). Although the specific set of reactions varies by compound, in many cases cytochrome P450 (CYP450) enzymes, carbonyl reductases, carboxylesterases, and other enzymes first alter xenobiotics through oxidation, reduction, and/or hydrolysis. Transferases then conjugate the resulting products with charged species like glucuronic acid and sulfate, forming polar derivatives that are more readily excreted via bile or urine (4).

Conflict of interest: Peter J. Turnbaugh is currently funded by Ethicon for work unrelated to this review article.

Reference information: J Clin Invest. 2014;124(10):4173-4181. doi:10.1172/JCI72335.
Owing to its diversity of enzymatic activities, the gut microbiome can metabolize many compounds in ways that humans cannot. Over 40 therapeutic and diet-derived bioactive compounds are known to undergo direct microbial modification (detailed reviews: refs. 5-7), but in nearly all cases the responsible microbial species or consortia remain unknown. Below we highlight examples of direct microbial metabolism of xenobiotics where linkages between properties of the microbial community and the relevant biochemical transformations have been elucidated. In addition, we provide examples of compounds whose activities appear to be altered through physical binding to microbial cells.

Microbial production of active compounds. The gut microbiome can convert inactive therapeutics (i.e., prodrugs) and dietary bioactives into their useful forms. For example, the prodrug sulfasalazine, prescribed for ulcerative colitis, consists of an antiinflammatory 5-aminosalicylic acid (5-ASA) molecule connected to a sulfapyridine molecule through an $\mathrm{N}-\mathrm{N}$ double bond (Figure 2). The drug remains inactive until it reaches the distal gut, where azoreductases encoded by the gut microbiome cleave the $\mathrm{N}-\mathrm{N}$ double bond to release active 5-ASA (8). Thus, fecal assays from conventional animals reflect complete conversion to constituent molecules, whereas germ-free or antibiotic-treated animals excrete the prodrug (8).

Similarly, fruits, vegetables, cereals, and coffee contain conjugated hydroxycinnamates, antioxidant and antiinflammatory compounds that are activated following microbial biotransformation (9-11). The active compounds - including caffeic acid, ferulic acid, and $p$-coumaric acid - are commonly present as ester conjugates in plants (Figure 2). Humans depend on the gut microbiome for the cinnamoyl esterases required to cleave these ester linkages, which explains why the caffeoyl, feruloyl, and $p$-coumaroyl groups exist in esterified form in the proximal gut, but as free acids in the colon (12, 13). Members of Bifidobacterium, Lactobacillus, and Escherichia in the human gut have been experimentally shown to hydrolyze esters of caffeic acid and ferulic acid (11); however, this activity is likely more widespread, as many bacteria and fungi produce cinnamoyl esterases (14). There are several additional examples of microbial metabolism of dietary compounds that yield bioactive molecules, 


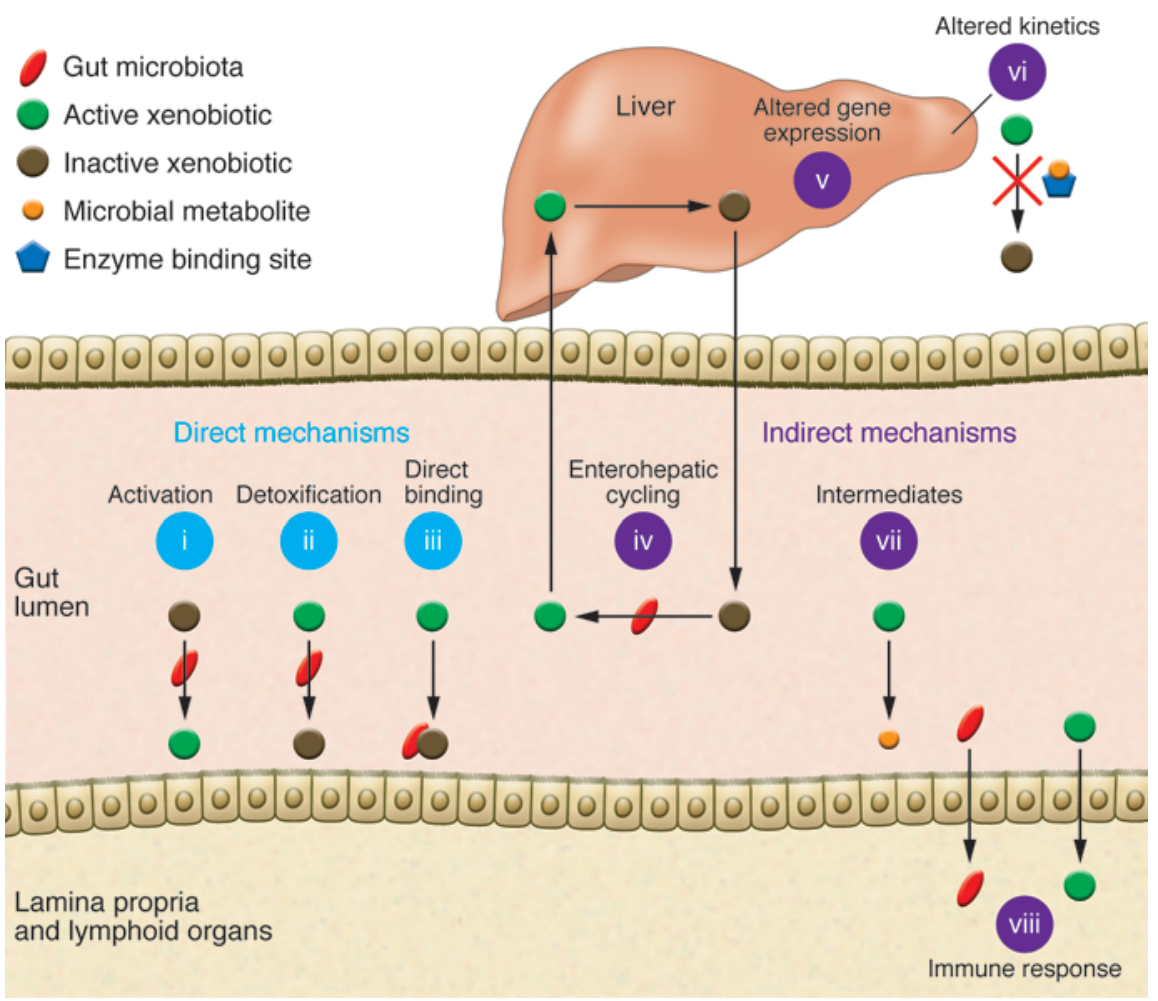

Figure 1. Gut microbial modulation of xenobiotic metabolism. Direct mechanisms include (i) production of active compounds, (ii) detoxification, and (iii) direct binding to bacterial cells. Indirect mechanisms involve microbial manipulation of host physiology. (iv) Microbial participation in enterohepatic cycling: (1) compounds are inactivated by conjugation in the liver; (2) the conjugated compound passes into bile and is excreted into the intestinal lumen; (3) microbial enzymes release the conjugate group, reactivating the compound; and (4) the active compound reenters circulation. (v) Altered expression of host genes involved in xenobiotic metabolism. (vi) Altered kinetics due to microbial metabolites competing with xenobiotics for enzyme binding sites. (vii) Microbial production of metabolic pathway intermediates. (viii) Stimulation of immune responses through translocation or inflammation.

including hydrolysis of cycasin into the carcinogenic glycoside methylazoxymethanol (15), production of the nonsteroidal estrogen equol from the soy-derived isoflavonoid daidzein (16), and liberation of aglycones with anticancer properties from anthocyanins (17).

Microbial detoxification of xenobiotics. The gut microbiome also encodes enzymes that detoxify xenobiotics, resulting in changes in efficacy and/or toxicity. The cardiac glycoside digoxin provides one of the few examples where a single bacterial species appears to be responsible for drug inactivation. Studies performed decades ago showed that digoxin is inactivated by the gut-residing Actinobacterium Eggerthella lenta (18), consistent with clinical reports of increased bioavailability after coadministration of digoxin and broad-spectrum antibiotics (19). E. lenta reduces a double bond in the $\alpha, \beta$-unsaturated lactone ring of the compound, producing a pharmacologically inactive metabolite, dihydrodigoxin, that is incapable of binding to its target cardiac receptor (Figure 2). We recently identified a cytochrome-encoding operon whose transcriptional activity is induced by digoxin and other cardiac glycosides. This "cardiac glycoside reductase" (cgr) operon is present in the type strain of E. lenta but missing in other nonreducing E. lenta strains (20). The abundance of the cgr operon predicts digoxin reduction by the human gut microbiome. Studies in mice monoassociated with reducing and nonreducing strains of $E$. lenta suggest that elevated levels of dietary protein can be used to suppress digoxin reduction, likely through inhibitory effects of elevated luminal arginine on cgr operon expression (21).

The gut microbiome also detoxifies dietary compounds. For example, many plants produce oxalate (22), a strong chelating agent that binds to $\mathrm{Ca}^{2+}$ and other free metal cations, contributing to hyperoxaluria, kidney stones, renal failure, and cardiac conduction disorders (23-25). Mammals lack the enzymes required to detoxify oxalate, relying instead on microbial biotransformation (ref. 26 and Figure 2). Three microbial enzymes are known to participate in the catabolism of oxalate: an oxalate:formate antiporter, which allows oxalate to enter the bacterial cell; formyl-CoA transferase, which converts oxalate to oxalyl-CoA; and oxalyl-CoA decarboxylase, which yields formyl-CoA. Oxalobacter formigenes is one of the key bacteria responsible for this reaction: (a) it can use oxalate as a sole carbon source (27); (b) lack of $O$. formigenes is associated with increased risk of hyperoxaluria and kidney stones (28); and (c) administration of $O$. formigenes reduces urinary oxalate concentrations $(29,30)$.

Direct binding to xenobiotics. Another potentially important but poorly understood interface between gut microbes and xenobiotics is through direct binding. The dopamine precursor drug levodopa (L-DOPA) is a widely prescribed treatment for Parkinson disease, whose clinical abnormalities are caused by dopamine depletion in the CNS. Unlike dopamine, orally administered L-DOPA can cross the blood-brain barrier, where it is decarboxylated into dopamine (31). It has long been observed that Parkinson patients have an increased risk of peptic ulceration (32), a condition now understood to result primarily from Helicobacter pylori-induced damage to the mucosa of the stomach and duodenum. Recent studies showed that eradication of $H$. pylori with antibiotics increases L-DOPA bioavailability in Parkinson patients, with a single antibiotic dose improving motor symptoms for 3 months or more (33). H. pylori may affect L-DOPA bioavailability by disrupting absorption at the duodenal mucosa (34), by producing ROS that inactivate the drug $(35,36)$, and via direct metabolism of L-DOPA (37). However, recent work suggests that $H$. pylori also compromises L-DOPA bioavailability through direct binding (Figure 2). In vitro incubations revealed 
Therapeutic example

Activation
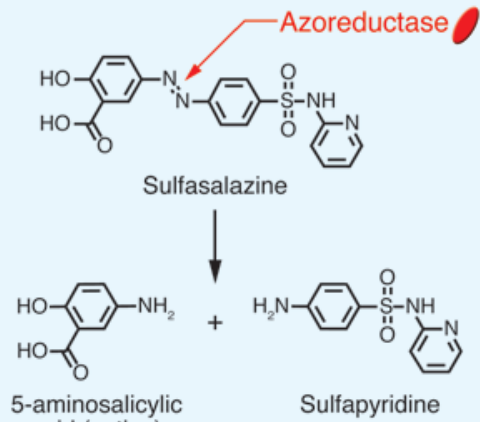
acid (active)

Detoxification
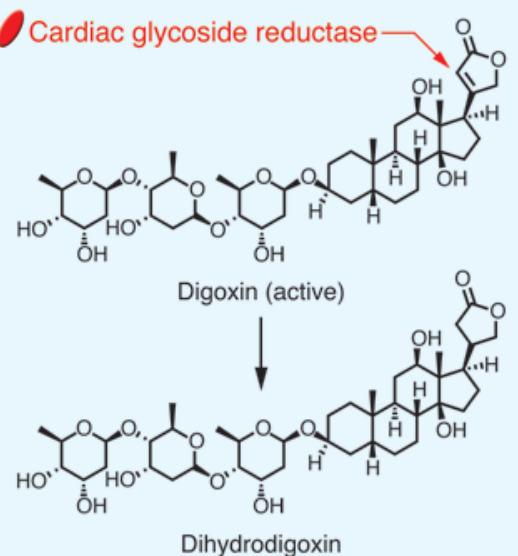

Direct binding

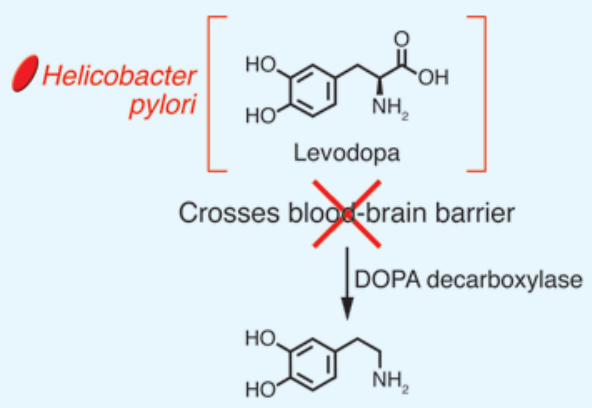

Dopamine (active)
Diet-derived example

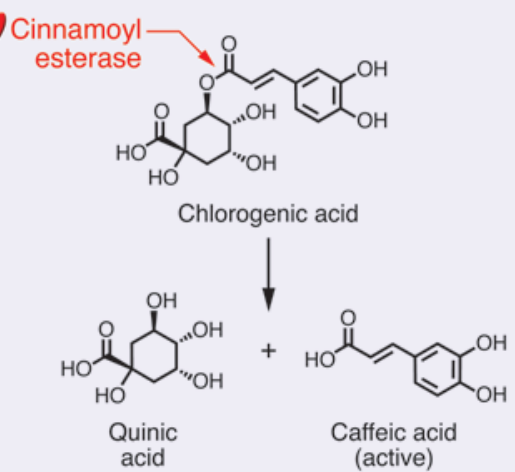

$$
\text { Formyl-CoA }
$$

Microbial activity

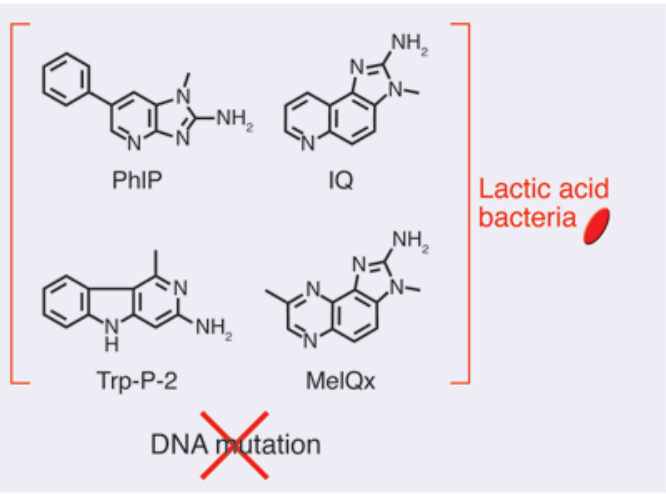

Figure 2. Mechanisms of direct microbial interference with select therapeutic and diet-derived compounds. See article text for detailed descriptions of these interactions.

a significant decrease in L-DOPA concentration upon exposure to $H$. pylori, with these exposed bacteria adhering less strongly to gastric epithelial cells because of binding of their outer membrane proteins by L-DOPA (38).

Direct binding by gut microbes may also have beneficial effects. Heterocyclic amines (HCAs) are formed during the charring of meat, poultry, and fish through the heat-catalyzed condensation of creatine, amino acids, and monosaccharides present in muscle tissue (39). Epidemiological studies have reported an association between intake of HCAs and increased risk of colorectal cancer $(40,41)$, with in vitro experiments and animal feeding trials supporting a causal link $(39,42)$. Multiple studies have reported that lactic acid bacteria present in the human gut and in fermented foods can directly bind to 2-amino-1-methyl-6-phenylimidazo [4,5$b$ ]pyridine (PhIP), 2-amino-3-methylimidazo[4,5-f quinoline (IQ), 2-amino-3,8-dimethylimidazo[4,5-f]quinoxaline (MeIQx), 3-amino-1-methyl-5H-pyrido(4,3-b)indole (Trp-P-2), and other important diet-derived mutagens (42-45), potentially preventing induction of DNA damage and preneoplastic lesions (ref. 46 and Figure 2). Additional studies are necessary to elucidate the range 


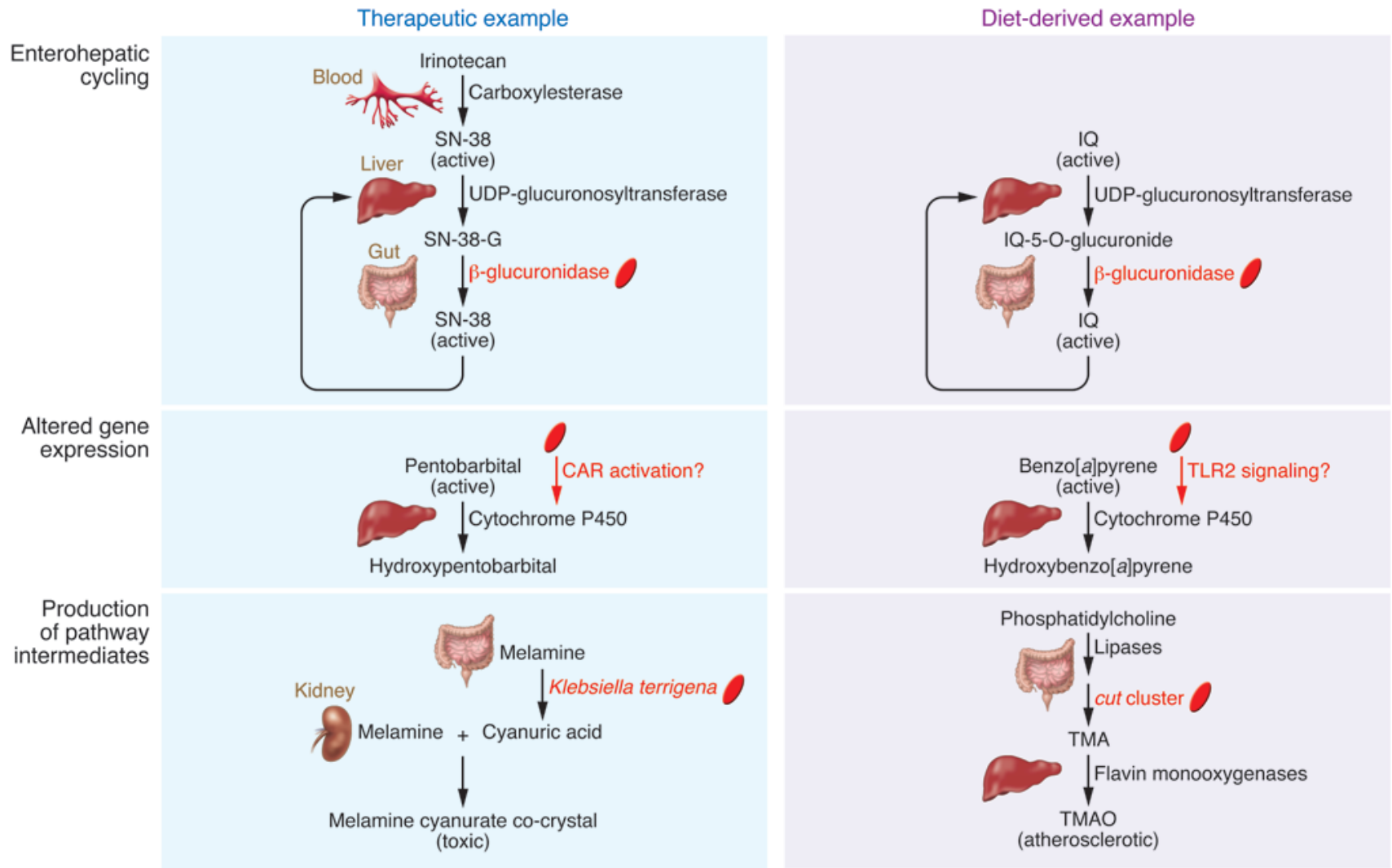

Therapeutic examples (no diet-derived examples yet known)

Altered kinetics
Stimulation of immune responses

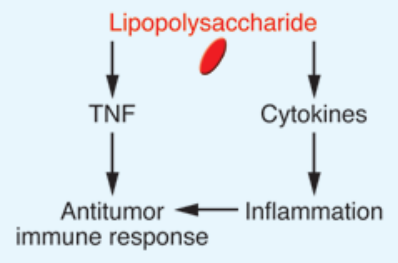

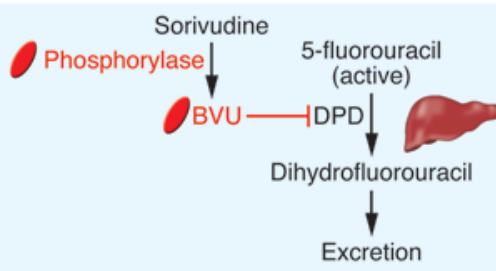

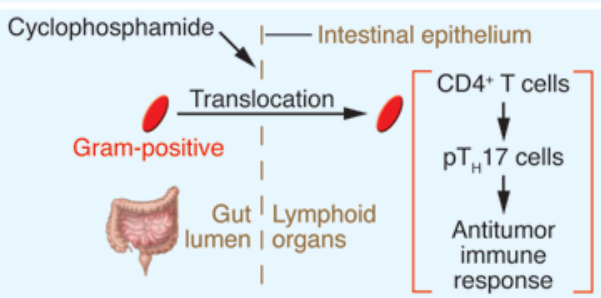

Microbial activity

Figure 3. Mechanisms of microbial modulation of host xenobiotic metabolism for select therapeutic and diet-derived compounds. See article text for detailed descriptions of these interactions.

of bacteria capable of directly binding to HCAs, the mechanisms of binding, the physiological effects on both the bacteria and the host, and the relevance of these phenomena to carcinogenesis.

\section{Indirect effects on xenobiotics via host-microbial interactions}

In addition to directly altering xenobiotics, recent work has emphasized that host xenobiotic metabolism is shaped by the gut microbiome. Below, we provide examples of the diverse mechanisms through which gut microbes are known to influence host xenobiotic metabolism. Further work in this area promises to elucidate long-standing questions about how we communicate with our associated microbes.

Enterohepatic cycling of xenobiotics. Xenobiotics are often conjugated to glucuronic acid by hepatic UDP-glucuronosyltransferase, producing a polar molecule that is readily excreted in bile (3). Once the conjugate enters the intestine, it is cleaved by microbial $\beta$-glucuronidase, often with deleterious consequences. For example, the colorectal cancer drug irinotecan (also known as CPT-11) is converted back into its cytotoxic form in the intestine (Figure 3), 
contributing to dose-limiting gastrointestinal side effects $(47,48)$. Broad-spectrum antibiotics and dietary fiber consumption have been proposed as general strategies that might reduce the toxicity of irinotecan $(49,50)$, but both of these interventions can have widespread impacts on the gut microbiota $(51,52)$. As an alternative and more targeted approach, researchers recently identified smallmolecule inhibitors of microbial $\beta$-glucuronidase that do not inhibit the mammalian ortholog or reduce the viability of either bacterial or human cells (53). Coadministration of irinotecan together with one of these inhibitors substantially reduced diarrheal symptoms in mice and protected gastrointestinal epithelial cells against inflammation (53). Remarkably, this same inhibitor has also been successfully applied to reduce enteropathy associated with the NSAID agents diclofenac, indomethacin, and ketoprofen, which likewise owe their side effects in part to $\beta$-glucuronidase $(54,55)$.

Enterohepatic cycling may also exacerbate harm from HCAs that are detoxified through hepatic glucuronidation, including abundant diet-derived compounds such as PhIP, IQ, and MeIQx (56). Studies of the carcinogenic and mutagenic compound IQ have repeatedly observed more DNA adducts and DNA damage in conventional mice versus their germ-free counterparts $(57,58)$. In addition, a recent study provided direct empirical support for a role of enterohepatic cycling in the genotoxicity of IQ (Figure 3). The genotoxicity of IQ was compared in gnotobiotic rats that were monoassociated with either Escherichia coli strain TG1 carrying the gene uidA, which codes for $\beta$-glucuronidase, or a uidAdeficient isogenic mutant (59). The presence of $\beta$-glucuronidase in the gut increased the genotoxicity of IQ threefold in the colon. Whereas the rate of urinary and fecal excretion of IQ reached a peak 24-48 hours after gavage in rats associated with the uidAdeficient strain, a second peak was observed after 144 hours in rats harboring the wild-type strain, suggesting that the compound reentered circulation. Further work is necessary to assess whether this process and its genotoxic consequences apply to other HCAs. Given the parallels between the elimination of irinotecan and NSAIDs and that of HCAs, it would be interesting to test whether the $\beta$-glucuronidase inhibitor that has proved beneficial for coadministration with irinotecan and NSAIDs also serves to reduce the genotoxicity of HCA compounds.

Altered host gene expression due to microbial colonization. Evidence is now emerging that the presence of a gut microbiota can alter key host enzymes involved in xenobiotic metabolism. For example, multiple studies have shown that the hepatic expression of CYP450 genes differs between the conventional and the germ-free state (60-62). Germ-free mice showed increased expression of P450 oxidoreductase, the sole electron donor for type II CYP450 enzymes, and increased expression of the constitutive androstane receptor (CAR), which serves as the master regulator of hepatic xenobiotic metabolism (62). These differences were functionally relevant; germ-free mice recovered 35\% faster from pentobarbital-induced anesthesia than conventional animals (Figure 3). One potential mechanism is that elevated levels of the CAR activators bilirubin, primary bile acids, and steroid hormones in germ-free animals may increase CAR expression and therefore enhance the rate of metabolism.

Microbial upregulation of host CYP450 enzymes may also confer protection against carcinogenic polycyclic aromatic hydrocarbons like benzo[a]pyrene, which is present in many plant and animal foods, but especially meats cooked over an open flame (63). A recent study presented initial evidence that intestinal detoxification of benzo[a]pyrene by CYP1A1 depends on TLR2, a receptor triggered by bacterial lipoproteins and other cell wall components (ref. 64 and Figure 3). Unlike wild-type mice, TLR2deficient $\left(T l r 2^{--}\right)$mice did not express CYP1A1 in the intestine upon exposure to benzo[a]pyrene. In addition, despite induction of CYP1A1 in the liver, $T l r 2^{-/-}$mice exhibited a reduced ability to clear benzo[a]pyrene from systemic circulation. Correspondingly, $\mathrm{Tl} \mathrm{r}^{-/-}$mice developed colon polyps after 21 days of dietary benzo[a]pyrene supplementation, a pathology absent in their wild-type counterparts. Although additional work is needed to clarify the mechanisms linking TLR2 to CYP1A1 expression, and to rule out alternative explanations such as altered patterns of benzo[a]pyrene absorption in $T l r 2^{-/-}$mice, these intriguing data raise the possibility that the gut microbiota modulates host xenobiotic metabolism in part through TLR2 signaling.

Production of pathway intermediates by gut microbes. Some of the most striking examples of host-microbial interactions come from metabolic pathways that share steps between host enzymes and those encoded by the gut microbiome. The recent melaminetainting scandal in China provides a disturbing example of how these intermediate microbial products can contribute to pathogenesis. In 2008, melamine-tainted milk products and pet foods led to an epidemic of kidney stones, acute kidney failure, and several deaths. The reasons were initially unclear, as melamine itself has very low acute toxicity. Biopsies from victims showed melamine urate precipitates in renal tubules $(65,66)$, a pathology known to be induced by coadministration of melamine and its structural analog cyanuric acid, which together form insoluble crystals (refs. 67,68 , and Figure 3). However, the source of the cyanuric acid was unclear, prompting researchers to test for microbial participation. In vitro and in vivo experiments showed that cyanuric acid is a metabolite formed during the degradation of melamine by gut bacteria such as the facultative anaerobe Klebsiella terrigena (69). Inoculation of melamine-fed rats with $K$. terrigena led to increased cyanuric acid concentration in the kidneys and renal toxicity, and antibiotic treatment was successful in reversing these effects (69).

An intermediate generated by the gut microbiome from dietary precursors has also been shown to contribute directly to cardiovascular disease (CVD), the leading cause of death and morbidity worldwide (70). A recent screen of plasma samples from a large clinical cohort found that risk of CVD was predicted by trimethylamine-containing (TMA-containing) metabolites of the dietary lipid phosphatidylcholine (also known as lecithin) that rely on the gut microbiome for their production (Figure 3). Dietary phosphatidylcholine is digested by intestinal lipases into a variety of choline-containing metabolites. Choline-containing metabolites that reach the colon undergo metabolism by microbial glycyl radical enzymes, labeled the choline utilization (cut) cluster (71), forming the intermediate gas TMA. TMA is absorbed and oxidized by hepatic flavin monooxygenases to form TMA $N$-oxide (TMAO), a metabolite linked to increased accumulation of cholesterol in macrophages, foam cell deposition, and atherosclerosis (72). Clinical studies have confirmed that TMAO production is also dependent on the gut microbiome in humans, with plasma TMAO levels suppressed during antibiotic treatment and restored upon 
antibiotic withdrawal (73). In this study, which followed roughly 4,000 patients over 3 years and controlled for risk factors, elevated plasma TMAO was associated with a higher risk of incurring an incident major adverse cardiac event.

Similarly, L-carnitine, a compound abundant in red meat, was recently shown to accelerate atherosclerosis in humans through the microbial production of TMA and subsequent hepatic production of TMAO (74). L-carnitine challenges administered following a week-long course of broad-spectrum antibiotics led to nearly complete suppression of TMAO production, with TMAO production returning following antibiotic withdrawal. Interestingly, dietinduced variation in gut microbial communities across subjects led to differences in TMAO production after an oral L-carnitine challenge, with omnivorous subjects showing increased capacity for TMAO formation compared with vegan or vegetarian subjects. Together, these findings are consistent with an obligatory role for the gut microbiome in TMAO formation from L-carnitine, and suggest that dietary history could potentially augment the risks of CVD associated with the intake of TMA precursors.

Microbial metabolites and xenobiotics compete for host enzymes. Metabolites generated by the gut microbiome can alter the efficacy or toxicity of xenobiotics by competing for host enzyme binding sites. For example, the common analgesic and antipyretic drug acetaminophen (also known as paracetamol) can be conjugated by 1 of 2 major pathways, $O$-sulfonation and glucuronidation. A small fraction of acetaminophen is transformed to toxic $\mathrm{N}$-acetylp-benzoquinone imine (NAPQI) by the CYP450 enzymes CYP2E1 and CYP3A4 (75). The toxicity of acetaminophen varies substantially across individuals $(76,77)$, and recent work suggests that microbial metabolites may be partly responsible. Clostridium difficile and other members of the gut microbiota produce $p$-cresol from the amino acid tyrosine (ref. 78). $p$-cresol is a substrate for the human liver enzyme SULT1A1, a cytosolic sulfotransferase that is also responsible for the $O$-sulfonation of acetaminophen (79). Thus $p$-cresol competes with acetaminophen for SULT1A1 binding sites as well as for availability of the sulfonate donor 3'-phosphoadenosine $5^{\prime}$-phosphosulfate (PAPS) (Figure 3). As a result, subjects with high pre-dose levels of $p$-cresol in urine excrete low post-dose fractions of acetaminophen sulfate versus acetaminophen glucuronide (79). Reduced capacity for acetaminophen in the $O$-sulfonation pathway due to occupancy of SULT1A1 by microbially produced $p$-cresol likely increases the production of NAPQI, resulting in a higher chance of hepatotoxicity. Such effects probably extend beyond acetaminophen, as a wide range of compounds are substrates for $O$-sulfonation via SULT1A1 (3).

A second, deadly example of the impact of competition for host enzyme binding sites on xenobiotic metabolism comes from the antiviral agent sorivudine. In 1993, 18 deaths and numerous cases of serious side effects were reported among Japanese cancer patients with the viral disease herpes zoster who were prescribed sorivudine on top of existing courses of the antitumor drug 5-fluorouracil (5-FU) or one of several 5-FU prodrugs. The combination therapy led to the toxic buildup of 5-FU due to inhibition of the 5 -FU catabolic enzyme dihydropyrimidine dehydrogenase (DPD) through irreversible binding by (E)-5-(2-bromovinyl)uracil (BVU), a microbial metabolite of sorivudine (ref. 80 and Figure 3). Germfree and antibiotic-treated rats evaded the deleterious effects of combination therapy, highlighting the critical role of the gut microbiota in this fatal drug-drug interaction. Sadly, these deaths might have been prevented, as the mechanisms of the microbial sorivudine-BVU conversion, the absorption of BVU in the intestine, and the observation that BVU irreversibly inhibits rat liver DPD resulting in enhanced plasma concentration of 5-FU after coinjection of 5-FU and BVU had already been reported in the literature (81).

Links between microbes, immunity, and xenobiotics. Recent studies of anticancer drugs demonstrate that the gut microbiome can also impact drug efficacy by stimulating the host immune system. For example, among tumor-bearing mice receiving CpG-oligonucleotide immunotherapy, antibiotic treatment reduced the production of TNF and proinflammatory cytokines, compromising the antitumor activity of myeloid-derived cells (ref. 82 and Figure 3). Gavage with LPS restored Tnf expression in germ-free mice, but not in mice that lack the TLR for LPS $\left(\mathrm{Tlr}^{-4^{-}}\right)$, suggesting that LPS production by Gram-negative bacteria primes the host immune system for CpG-oligonucleotide immunotherapy. Confirming this pattern, Tnf expression was positively correlated with the abundance of the Gram-negative genus Alistipes, and inoculation by Alistipes after antibiotic treatment rescued TNF production (82).

Gram-positive bacteria have also been credited with improving cancer drug efficacy. Intestinal permeability caused by the anticancer prodrug cyclophosphamide led to significant translocation of Gram-positive bacterial species (primarily Lactobacillus johnsonii and Enterococcus hirae) out of the gut and into mesenteric lymph nodes and spleen (ref. 83 and Figure 3). Translocated bacteria stimulated the differentiation of pathogenic Th17 $\left(\mathrm{pT}_{\mathrm{H}} 17\right)$ cells from naive $\mathrm{CD} 4^{+} \mathrm{T}$ cells in vitro and in vivo, whereas control bacteria and purified LPS did not induce a response. Germ-free mice or mice treated with vancomycin, an antibiotic that primarily targets Gram-positive bacteria, exhibited a reduction in $\mathrm{pT}_{\mathrm{H}} 17$ responses that inhibited the antitumor effects of cyclophosphamide, with adoptive transfer of $\mathrm{pT}_{\mathrm{H}} 17$ cells into vancomycin-treated mice rescuing these effects.

Together, such studies suggest that the common practice of administering antibiotics with chemotherapy, typically done to offset low white blood cell counts, carries previously unappreciated therapeutic risks. Additional work is necessary to determine the relative contributions of direct stimulation of the immune system by bacterial antigens and indirect effects due to microbial metabolism of the administered compounds and/or microbial modifications of host xenobiotic metabolism. It will also be important to test whether interindividual variations in microbial community structure and function contribute to clinically relevant changes in drug response.

\section{Prospectus}

Over the past few decades, elegant experiments exploiting in vitro and ex vivo incubation, antibiotic supplementation, and the comparison of conventional and germ-free animals established an appreciation for the vast array of xenobiotic biotransformations catalyzed by the gut microbiome. Yet only rarely could these methods achieve mechanistic insight sufficient to predict how a given individual would respond to a xenobiotic agent. Recent advances in metagenomics, metabolomics, bacterial genetics, biochemistry, and microfluidics have dramatically expanded our ability to characterize the composition, transcriptional activities, 
and metabolic products of the human microbiome. Used together, these methods have already delivered unprecedented, predictive views of host response and strategies to manipulate this response favorably. Early examples include the use of the $\mathrm{cgr}$ operon to predict the inactivation of the cardiac drug digoxin (20), as well as the development of a targeted microbial $\beta$-glucuronidase inhibitor that minimizes side effects of both the cancer drug irinotecan (53) and several NSAIDs $(54,55)$. Pursuing a mechanistic understanding of the direct and indirect manipulation of xenobiotics by the gut microbiome, and their interactions with host and environmental factors, could ultimately lead to proactive exploitation of these abilities for therapeutic benefit.

There are many challenges ahead. The inherent complexity of host-microbial interactions has led Nicholson and Wilson (84) to compare xenobiotic metabolism to pachinko (Japanese pinball), in which the outcome is determined by the probabilistic movement and collisions of myriad host and microbial elements. In their model, the fate of a ball (xenobiotic) with a particular size and shape (biochemical properties) will depend on the distribution of pins (human and microbial enzymes) that determine its route through the machine (body) and the likely point at which it exits (excretion). The probabilistic model is further complicated in that the number and position of pins are continually changing, responsive to interactions between host genetics, the composition and function of the gut microbial community, and numerous environmental factors.

Although some elements of this complex interplay cannot be easily manipulated, several future innovations could help to increase the probability of favorable paths. First, the development of microbiome screening assays could help doctors assess the compatibility between individual patients and candidate drugs. Second, the global metabolic potential of the gut microbiome could be manipulated through dietary $(51,85)$, antibiotic $(52,86)$, probiotic/ prebiotic/synbiotic $(87,88)$, and/or transplantation (89) interventions to increase the probability of a favorable outcome. Third, compounds that target particular functions of the gut microbiome could be developed to increase favorable metabolic responses or inhibit unfavorable ones, as exemplified by the microbial $\beta$-glucuronidase inhibitor developed by Wallace and colleagues (53). Fourth, leveraging the ability of bacteria to exchange capabilities via lateral gene transfer, probiotic species could be engineered to deliver specific metabolic capabilities into the microbiome. A natural example of this phenomenon is the transfer of porphyran-degrading capability from the marine bacterium Zobellia galactanivorans to the gut bacterium Bacteroides plebeius resident in Japanese populations with a history of consuming porphyran-rich seaweed (90). Finally, simple deliberate efforts to consider host-microbial metabolic interactions in the development and administration of xenobiotics could yield lifesaving benefits, as illustrated by the tragic case of sorivudine, whose fatal drug-drug interaction with 5-FU could have been predicted on the basis of knowledge at the time (81).

Fundamental questions concerning the role of the gut microbiome remain unanswered for the majority of therapeutic and diet-derived xenobiotics, and for microbial metabolism in general. What are the microbial taxa and molecular mechanisms involved? Which metabolic activities are ubiquitous and which vary by host? How do host and environmental factors affect microbial metabolism? To what extent is microbial metabolism stable, transmissible, and resilient to perturbation? Do past exposures to a given compound influence future microbial response? What selective forces have shaped the evolution of microbial enzymes for xenobiotic metabolism, and what is the range of accessible natural and foreign substrates? Ultimately, answering such questions could be the key to a more comprehensive view of human biology and a more predictive and personalized approach to medicine.

\section{Acknowledgments}

We sincerely apologize to our many outstanding colleagues whose work was left out because of space limitations; a more comprehensive review on this subject is still needed. We thank Emily Balskus, Nitzan Koppel, Kristen Seim, and the 3 anonymous reviewers for helpful comments on the manuscript. This work was supported by the NIH (1F32DK101154, P50GM068763) and the Bauer Fellows program at Harvard University.

Address correspondence to: Peter J. Turnbaugh, FAS Center for Systems Biology, Harvard University, 52 Oxford Street, Cambridge, Massachusetts 02138, USA. Phone: 617.384.9238; E-mail: pturnbaugh@fas.harvard.edu.
1. Backhed F, Ley RE, Sonnenburg JL, Peterson DA, Gordon JI. Host-bacterial mutualism in the human intestine. Science. 2005;307(5717):1915-1920.

2. Qin J, et al. A human gut microbial gene catalogue established by metagenomic sequencing. Nature. 2010;464(7285):59-65.

3. Klaassen CD. Casarett \& Doull's Toxicology: The Basic Science of Poisons. 8th ed. New York, New York, USA: McGraw-Hill; 2013.

4. Finkel R, Cubeddu LX, Clark MA. Pharmacology. 4th ed. Baltimore, Maryland, USA: Lippincott Williams \& Wilkins; 2009.

5. Sousa T, Paterson R, Moore V, Carlsson A, Abrahamsson B, Basit AW. The gastrointestinal microbiota as a site for the biotransformation of drugs. Int J Pharm. 2008;363(1-2):1-25.

6. Saad R, Rizkallah MR, Aziz RK. Gut pharmacomicrobiomics: the tip of an iceberg of complex interactions between drugs and gut-associated microbes. Gut Pathogens. 2012;4(1):16.

7. Haiser HJ, Turnbaugh PJ. Developing a metagenomic view of xenobiotic metabolism. Pharmacol Res. 2013;69(1):21-31.

8. Peppercorn MA, Goldman P. The role of intestinal bacteria in the metabolism of salicylazosulfapyridine. J Pharm Exp Ther. 1972;181(3):555-562.

9. Laparra JM, Sanz Y. Interactions of gut microbiota with functional food components and nutraceuticals. Pharmacol Res. 2010;61(3):219-225.

10. Peppercorn MA, Goldman P. Caffeic acid metabolism by gnotobiotic rats and their intestinal bacteria. Proc Natl Acad Sci US A. 1972;69(6):1413-1415

11. Couteau D, McCartney AL, Gibson GR, Williamson G, Faulds CB. Isolation and characterization of human colonic bacteria able to hydrolyse chlorogenic acid. J Appl Microbiol. 2001;90(6):873-881.

12. Plumb GW, Garcia-Conesa MT, Kroon PA, Rhodes M, Ridley S, Williamson G. Metabolism of chlorogenic acid by human plasma, liver, intestine and gut microflora. J Sci Food Agr. 1999;79(3):390-392.

13. Buchanan CJ, Wallace G, Fry SC, Eastwood MA. In vivo release of $14 \mathrm{C}$-labelled phenolic groups from intact dietary spinach cell walls during passage through the rat intestine. J Sci Food Agr. 1996;71(4):459-469.

14. Donaghy J, Kelly PF, McKay AM. Detection of ferulic acid esterase production by Bacillus spp. and lactobacilli. Appl Microbiol Biotechnol. 1998;50(2):257-260.

15. Spatz M, Smith DW, McDaniel EG, Laqueur GL. Role of intestinal microorganisms in determining cycasin toxicity. P Soc Exp Biol Med. 1967;124(3):691-697.

16. Tsuji H, Moriyama K, Nomoto K, Akaza H. Identification of an enzyme system for daidzein-toequol conversion in Slackia sp. strain natts. Appl 
Environ Microbiol. 2012;78(4):1228-1236.

17. Selma MV, Espin JC, Tomas-Barberan FA. Interaction between phenolics and gut microbiota: role in human health. J Agr Food Chem. 2009;57(15):6485-6501.

18. Dobkin JF, Saha JR, Butler VP, Neu HC, Lindenbaum J. Digoxin-inactivating bacteria: identification in human gut flora. Science. 1983;220(4594):325-327.

19. Lindenbaum J, Rund DG, Butler VP, Tse-Eng $\mathrm{D}$, Saha JR. Inactivation of digoxin by the gut flora: reversal by antibiotic therapy. $\mathrm{NEngl} \mathrm{J} \mathrm{Med.}$ 1981;305(14):789-794.

20. Haiser HJ, Gootenberg DB, Chatman K, Sirasani G, Balskus EP, Turnbaugh PJ. Predicting and manipulating cardiac drug inactivation by the human gut bacterium Eggerthella lenta. Science. 2013;341(6143):295-298.

21. Haiser HJ, Seim KL, Balskus EP, Turnbaugh PJ. Mechanistic insight into digoxin inactivation by Eggerthella lenta augments our understanding of its pharmacokinetics. Gut Microbes. 2014;5(2):233-238.

22. Franceschi VR, Nakata PA. Calcium oxalate in plants: formation and function. Annu Rev Plant Biol. 2005;56(1):41-71.

23. Williams HE, Smith LH. Disorders of oxalate metabolism. Am JMed. 1968;45(5):715-735.

24. James LF. Oxalate toxicosis. Clin Toxicol. 1972;5(2):231-243.

25. Rodby RA, Tyszka TS, Williams JW. Reversal of cardiac dysfunction secondary to type 1 primary hyperoxaluria after combined liver-kidney transplantation. Am JMed.1991;90(4):498-504.

26. Miller AW, Dearing D. The metabolic and ecological interactions of oxalate-degrading bacteria in the mammalian gut. Pathogens. 2013;2(4):636-652.

27. Allison MJ, Cook HM, Milne DB, Gallagher $\mathrm{S}$, Clayman RV. Oxalate degradation by gastrointestinal bacteria from humans. J Nutr. 1986;116(3):455-460.

28. Stewart CS, Duncan SH, Cave DR. Oxalobacter formigenes and its role in oxalate metabolism in the human gut. FEMS Microbiol Lett. 2004;230(1):1-7.

29. Hatch M, Gjymishka A, Salido EC, Allison MJ, Freel RW. Enteric oxalate elimination is induced and oxalate is normalized in a mouse model of primary hyperoxaluria following intestinal colonization with Oxalobacter. Am J Physiol Gastrointest Liver Physiol. 2011;300(3):G461-G469.

30. Hoppe B, et al. Oxalobacter formigenes: a potential tool for the treatment of primary hyperoxaluria type I. Kidney Int. 2006;70(7):1305-1311.

31. Goole J, Amighi K. Levodopa delivery systems for the treatment of Parkinson's disease: an overview. Int J Pharm. 2009;380(1-2):1-15.

32. Strang RR. The association of gastro-duodenal ulceration with Parkinson's disease. Med J Aust. 1965;1(23):842-843.

33. Pierantozzi M, Pietroiusti A, Brusa L, et al. Helicobacter pylori eradication and L-dopa absorption in patients with $\mathrm{PD}$ and motor fluctuations. Neurology. 2006;66(12):1824-1829.

34. Hamlet A, Thoreson AC, Nilsson O, Svennerholm AM, Olbe L. Duodenal Helicobacter pylori infection differs in cagA genotype between asymptomatic subjects and patients with duodenal ulcers. Gastroenterology. 1999;116(2):259-268.

35. Davies GR, et al. Helicobacter pylori stimulates antral mucosal reactive oxygen metabolite production in-vivo. Gut. 1994;35(2):179-185.

36. Kankkunen T, et al. Improved stability and release control of levodopa and metaraminol using ion-exchange fibers and transdermal iontophoresis. Eur J Pharm Sci. 2002;16(4-5):273-280.

37. Goldin BR, Peppercorn MA, Goldman P. Contributions of host and intestinal microflora in the metabolism of L-dopa by the rat.J Pharm Exp Ther. 1973;186(1):160-166.

38. Niehues M, Hensel A. In-vitro interaction of L-dopa with bacterial adhesins of Helicobacter pylori: an explanation for clinicial differences in bioavailability? J Pharm Pharmacol. 2009;61(10):1303-1307.

39. Sugimura T, Wakabayashi K, Nakagama H, Nagao M. Heterocyclic amines: mutagens/carcinogens produced during cooking of meat and fish. Cancer Sci. 2004;95(4):290-299.

40. Cross AJ, et al. A large prospective study of meat consumption and colorectal cancer risk: an investigation of potential mechanisms underlying this association. Cancer Res. 2010;70(6):2406-2414.

41. Rohrmann S, Hermann S, Linseisen J. Heterocyclic aromatic amine intake increases colorectal adenoma risk: findings from a prospective European cohort study. Am J Clin Nutr. 2009;89(5):1418-1424.

42. Orrhage K, Sillerström E, Gustafsson JÅ, Nord CE, Rafter J. Binding of mutagenic heterocyclic amines by intestinal and lactic acid bacteria. Mutat Res. 1994;311(2):239-248.

43. Turbic A, Ahokas JT, Haskard CA. Selective in vitro binding of dietary mutagens, individually or in combination, by lactic acid bacteria. Food Addit Contam. 2002;19(2):144-152.

44. Morotomi M, Mutai M. In vitro binding of potent mutagenic pyrolysates to intestinal bacteria. J Natl Cancer Inst. 1986;77(1):195-201.

45. Knasmuller S, Steinkellner H, Hirschl AM, Rabot S, Nobis EC, Kassie F. Impact of bacteria in dairy products and of the intestinal microflora on the genotoxic and carcinogenic effects of heterocyclic aromatic amines. Mutat Res. 2001; 480-481(1):129-138.

46. Zsivkovits M, et al. Prevention of heterocyclic amine-induced DNA damage in colon and liver of rats by different lactobacillus strains. Carcinogenesis. 2003;24(12):1913-1918.

47. Takasuna $\mathrm{K}$, et al. Inhibition of intestinal microflora beta-glucuronidase modifies the distribution of the active metabolite of the antitumor agent, irinotecan hydrochloride (CPT-11) in rats. Cancer Chemoth Pharm. 1998;42(4):280-286.

48. Gupta E, Lestingi TM, Mick R, Ramirez J, Vokes EE, Ratain MJ. Metabolic fate of irinotecan in humans: correlation of glucuronidation with diarrhea. Cancer Res. 1994;54(14):3723-3725.

49. Lin XB, et al. The role of intestinal microbiota in development of irinotecan toxicity and toxicity reduction through dietary fibres in rats. $P L O S$ One. 2014;9(1):e83644.

50. Kehrer DF, et al. Modulation of irinotecan-induced diarrhea by cotreatment with neomycin in cancer patients. Clin Cancer Res. 2001;7(5):1136-1141.

51. David LA, et al. Diet rapidly and reproduc- ibly alters the human gut microbiome. Nature. 2014;505(7484):559-563.

52. Dethlefsen L, Relman DA. Incomplete recovery and individualized responses of the human distal gut microbiota to repeated antibiotic perturbation. Proc Natl Acad Sci U S A. 2011;108(suppl 1):4554-4561.

53. Wallace BD, et al. Alleviating cancer drug toxicity by inhibiting a bacterial enzyme. Science. 2010;330(6005):831-835.

54. Saitta KS, Zhang C, Lee KK, Fujimoto K, Redinbo MR, Boelsterli UA. Bacterial $\beta$-glucuronidase inhibition protects mice against enteropathy induced by indomethacin, ketoprofen or diclofenac: mode of action and pharmacokinetics. Xenobiotica. 2013;44(1):28-35.

55. LoGuidice A, Wallace BD, Bendel L, Redinbo MR, Boelsterli UA. Pharmacologic targeting of bacterial $\beta$-glucuronidase alleviates nonsteroidal anti-inflammatory drug-induced enteropathy in mice. J Pharm Exp Ther. 2012;341(2):447-454.

56 . Kaderlik KR, et al. Glucuronidation of N-hydroxy heterocyclic amines by human and rat liver microsomes. Carcinogenesis. 1994;15(8):1695-1701.

57. Hirayama K, Baranczewski P, Akerlund JE, Midtvedt T, Moller L, Rafter J. Effects of human intestinal flora on mutagenicity of and DNA adduct formation from food and environmental mutagens. Carcinogenesis. 2000;21(11):2105-2111.

58. Kassie F, Rabot S, Kundi M, Chabicovsky M, Qin $\mathrm{HM}$, Knasmuller S. Intestinal microflora plays a crucial role in the genotoxicity of the cooked food mutagen 2-amino-3-methylimidazo[4,5-f]quinoline (IQ). Carcinogenesis. 2001;22(10):1721-1725.

59 . Humblot $C$, et al. $\beta$-glucuronidase in human intestinal microbiota is necessary for the colonic genotoxicity of the food-borne carcinogen 2-amino-3-methylimidazo[4,5-f]quinoline in rats. Carcinogenesis. 2007;28(11):2419-2425.

60. Toda $\mathrm{T}$, et al. Intestinal flora induces the expression of Cyp3a in the mouse liver. Xenobiotica. 2009;39(4):323-334.

61. Claus SP, et al. Colonization-induced hostgut microbial metabolic interaction. MBio. 2011;2(2):e00271-10.

62. Bjorkholm B, Bok CM, Lundin A, Rafter J, Hibberd ML, Pettersson S. Intestinal microbiota regulate xenobiotic metabolism in the liver. PLoS One. 2009;4(9):e6958.

63. Phillips DH. Polycyclic aromatic hydrocarbons in the diet. Mutat Res. 1999;443(1-2):139-147.

64. Do KN, Fink LN, Jensen TE, Gautier L, Parlesak A. TLR2 controls intestinal carcinogen detoxification by CYP1A1. PLoS One. 2011;7(3):e32309.

65. Chang H, Shi XF, Shen W, Wang W, Yue ZJ. Characterization of melamine-associated urinary stones in children with consumption of melamine-contaminated infant formula. Clin Chim Acta. 2012;413(11-12):985-991.

66. Sun N, et al. Diagnosis and treatment of melamine-associated urinary calculus complicated with acute renal failure in infants and young children. Chin Med J. 2009;122(3):245-251.

67. Kobayashi $\mathrm{T}$, et al. The mechanism of renal stone formation and renal failure induced by administration of melamine and cyanuric acid. Urol Res. 2010;38(2):117-125.

68. Xie GX, et al. Metabonomic evaluation of melamine-induced acute renal toxicity in rats. 
J Proteome Res. 2010;9(1):125-133.

69. Zheng XJ, et al. Melamine-induced renal toxicity is mediated by the gut microbiota. Sci Transl Med. 2013;5(172):172ra122.

70. Mathers CD, Loncar D. Projections of global mortality and burden of disease from 2002 to 2030. PLoS Med. 2006;3(11):e442.

71. Craciun S, Balskus EP. Microbial conversion of choline to trimethylamine requires a glycyl radical enzyme. Proc Natl Acad Sci U S A. 2012;109(52):21307-21312.

72. Wang Z, et al. Gut flora metabolism of phosphatidylcholine promotes cardiovascular disease. Nature. 2011;472(7341):57-63.

73. Tang WHW, et al. Intestinal microbial metabolism of phosphatidylcholine and cardiovascular risk. N Engl J Med. 2013;368(17):1575-1584.

74. Koeth RA, et al. Intestinal microbiota metabolism of L-carnitine, a nutrient in red meat, promotes atherosclerosis. Nat Med. 2013;19(5):576-585.

75. Laine JE, Auriola S, Pasanen M, Juvonen RO. Acetaminophen bioactivation by human cytochome $\mathrm{P} 450$ enzymes and animal microsomes. Xenobiotica. 2009;39(1):11-21.

76. Harrill AH, et al. Mouse population-guided resequencing reveals that variants in CD44 contribute to acetaminophen-induced liver injury in humans. Genome Res. 2009;19(9):1507-1515.
77. Watkins PB, et al. Aminotransferase elevations in healthy adults receiving 4 grams of acetaminophen daily - a randomized controlled trial. J Am Med Assoc. 2006;296(1):87-93.

78. Selmer T, Andrei PI. p-Hydroxyphenylacetate decarboxylase from Clostridium difficile: a novel glycyl radical enzyme catalysing the formation of p-cresol. Eur J Biochem. 2001;268(5):1363-1372.

79. Clayton TA, Baker D, Lindon JC, Everett JR, Nicholson JK. Pharmacometabonomic identification of a significant host-microbiome metabolic interaction affecting human drug metabolism. Proc Natl Acad Sci U S A. 2009;106(34):14728-14733.

80. Nakayama H, Kinouchi T, Kataoka K, Akimoto S, Matsuda Y, Ohnishi Y. Intestinal anaerobic bacteria hydrolyse sorivudine, producing the high blood concentration of 5-(E)-(2-bromovinyl)uracil that increases the level and toxicity of 5-fluorouracil. Pharmacogenetics. 1997;7(1):35-43.

81. Okuda H, et al. Lethal drug interactions of sorivudine, a new antiviral drug, with oral 5-fluorouracil prodrugs. Drug Metab Dispos. 1997;25(2):270-273.

82. Iida $\mathrm{N}$, et al. Commensal bacteria control cancer response to therapy by modulating the tumor microenvironment. Science. 2013;342(6161):967-970.

83. Viaud S, et al. The intestinal microbiota modulates the anticancer immune effects of cyclo- phosphamide. Science. 2013;342(6161):971-976.

84. Nicholson JK, Wilson ID. Understanding 'global' systems biology: metabonomics and the continuum of metabolism. Nat Rev Drug Discov. 2003;2(1):668-676.

85. Faith JJ, McNulty NP, Rey FE, Gordon JI. Predicting a human gut microbiota's response to diet in gnotobiotic mice. Science. 2011;333(6038):101-104.

86. Dethlefsen L, Huse S, Sogin ML, Relman DA. The pervasive effects of an antibiotic on the human gut microbiota, as revealed by deep 16S rRNA sequencing. PLoS Biol. 2008;6(11):2383-2400.

87. McNulty NP, et al. The impact of a consortium of fermented milk strains on the gut microbiome of gnotobiotic mice and monozygotic twins. Sci Transl Med. 2011;3(106):106ra106.

88. Delzenne NM, Neyrinck AM, Backhed F, Cani PD. Targeting gut microbiota in obesity: effects of prebiotics and probiotics. Nat Rev Endocrinol. 2011;7(11):639-646.

89. Borody TJ, Khoruts A. Fecal microbiota transplantation and emerging applications. Nat Rev Gastroenterol Hepatol. 2012;9(2):88-96.

90. Hehemann J-H, Correc G, Barbeyron T, Helbert W, Czjzek M, Michel G. Transfer of carbohydrate-active enzymes from marine bacteria to Japanese gut microbiota. Nature. 2010;464(7290):908-912. 